\title{
Efficacy of Low Dose Chemoprophylaxis for Coccidioidomycosis Infection in Liver Transplant Recipients
}

\author{
Shahid Habib ${ }^{\mathrm{a}, \mathrm{j}}$, Razan A. El Ramahi ${ }^{\mathrm{b}}$, Scott Rosen ${ }^{\mathrm{c}}$, Sumaya Farran ${ }^{\mathrm{d}}$, \\ Jamilah Shubeilate, Courtney Walker ${ }^{\mathrm{f}}$, Mariana Casalg, \\ Tirdad Zangeneh ${ }^{\mathrm{h}, \mathrm{i}}$
}

\begin{abstract}
Background: Coccidioidomycosis (CM) infections among transplant recipients result in significant morbidity and mortality. The goal of our study was to establish the efficacy of low dose (LD) versus standard dose (LD, $50 \mathrm{mg}$ daily) fluconazole in preventing CM infection.

Methods: This was a retrospective study utilizing electronic medical records of liver transplant recipients at the University of Arizona. The primary end point was post-transplant $\mathrm{CM}$ status, such as infection, complications and survival.

Results: We detected a statistically significant correlation between positive pre-transplant status and positive post-transplant status (hazards ratio: 8.25 (95\% confidence interval: 1.028 - 66.192)). There was a trend towards improved survival in patients who had a positive posttransplant CM status in the SD group versus LD group (90.9\% versus $81.3 \%$ ), although not statistically significant.
\end{abstract}

Conclusion: The risk of $\mathrm{CM}$ infection among transplant recipients in the absence of prophylaxis is associated with high morbidity and

Manuscript submitted April 15, 2019, accepted May 13, 2019

aLiver Institute PLLC, 2830 N. Swan Rd, Suite 180, Tucson, AZ 85712, USA banner University Medical Center, 1501 North Campbell Avenue, Tucson, AZ 85724, USA

'Skyline Medical Center, 3441 Dickerson Pike, Nashville, TN 37207, USA

dBanner University Medical Center, 1501 North Campbell Avenue, Tucson, AZ 85724, USA

eBMJ Medical Group, Boone Hospital Center, 1605 East Broadway, Suite 110, Columbia, MO 65201, USA

fUniversity of Utah Health Care, 30 North 1900 East, SOM 4R118, SLC, UT 84132, USA

${ }^{\mathrm{g}}$ Arizona Department of Health Services, 400 W. Congress Street, Suite 116, Tucson, AZ 85701, USA

hUniversity of Arizona, Banner University Medical Center, 1502 North Campbell Avenue, Tucson, AZ 85724, USA

iValley Fever Center for Excellence, 1656 E Mabel St, Tucson, AZ 85724, USA

${ }^{j}$ Corresponding Author: Shahid Habib, Liver Institute PLLC, 2830 N. Swan Rd, Tucson, AZ 85712, USA. Email: shabib@liverinstitutepllc.org

doi: https://doi.org/10.14740/gr1182 mortality. We currently use SD fluconazole as universal prophylaxis in all transplant recipients despite not establishing statistical significance between LD and SD. We believe that the survival trend detected may have not reached statistical significance due to low power impact. Since the standardization of SD prophylaxis at our institution, we have not diagnosed further new post-transplant CM infections.

Keywords: Coccidioidomycosis; Fungal infection; Prophylaxis; Liver transplant

\section{Introduction}

The dimorphic fungi, Coccidioides immitis/posadasii, are endemic to Southwestern United States, Northern Mexico, Central America and South America. Coccidioidomycosis (CM), also known as San Joaquin Valley fever, is associated with infections among immunocompromised patients including transplant recipients and can result in significant morbidity and mortality in this population. In 2015, there were 7,622 cases of $\mathrm{CM}$ reported in Arizona [1]. The risk of infection among solidorgan transplant (SOT) recipients in endemic regions in the absence of prophylaxis during the early transplantation years has been reported to be $7-9 \%$ with a high rate of dissemination of up to $75 \%$ and mortality of $63 \%$ [2]. In most cases, infection is thought to be a result of reactivation of prior exposure [2] and the majority of these infections occur within the first year after transplantation. Although there are no established guidelines, the Infectious Diseases Society of America (IDSA) does recommend prophylaxis for special at-risk populations [3] and most transplant centers have protocols for prophylaxis for transplant recipients. Transplant recipients at the University of Arizona previously received fluconazole $50 \mathrm{mg}$ daily as prophylaxis for an indefinite period of time. The efficacy of this practice had not been established by major studies. Transplant centers in the endemic area use different approaches for the prevention of $\mathrm{CM}$ in their recipients. Blair et al recently reported data that universal prophylaxis with fluconazole 200 $\mathrm{mg}$ daily for 12 months after liver transplantation was effective for those residing in endemic regions [4]. The aims of our study were to 1) establish the risk of new CM infection or reac- 
tivation in liver transplant recipients living in Arizona, receiving prophylaxis with fluconazole dose of less than $200 \mathrm{mg} /$ day versus $200 \mathrm{mg} /$ day, 2) assess the risk of infection among groups who received prophylaxis for less than 6 months, between 6 and 12 months and more than 12 months and 3) establish CM prophylaxis guidelines in our institution for liver transplant recipients.

\section{Materials and Methods}

\section{Study design}

This was a retrospective study where we reviewed the electronic medical records (EMR) of liver transplant recipients at the University of Arizona. Inclusion criteria considered 1) all adult and pediatric patients who underwent liver transplantation from January 1, 1998 through May 31, 2012 and 2) all patients who had CM serology testing performed during this period. Exclusion criteria considered 1) patients who received treatment dose antifungal therapy at the time of their transplantation and/or 2) patients who had incomplete data regarding prophylaxis status in the charts. Study participants were categorized into two groups according to the prophylaxis dose: 1) low dose (LD) prophylaxis group: $<200 \mathrm{mg}$ per day of fluconazole ( $<6 \mathrm{mg} / \mathrm{kg} /$ day for pediatric patients) and 2) standard dose (SD) prophylaxis group: $200 \mathrm{mg}$ per day of fluconazole $(6 \mathrm{mg} / \mathrm{kg} /$ day for pediatric patients). The primary end point of the study was the post-transplant CM status, such as infection, complications and survival. The study was conducted in compliance with the ethical standards of the responsible institution on human subjects as well as with the Helsinki Declaration. The University of Arizona Institutional Review Board (IRB) approved the study.

\section{Data collection}

Laboratory results data as well as progress notes, admission history, surgical reports, discharge summaries and scanned medical documents in our inpatient and outpatient EMR were searched. Data collected included demographic information, etiology of liver failure, other comorbid conditions, immunosuppressive regimen, rejection episodes and treatment, and donor details when available.

Data pertaining to study key elements included pre-transplantation CM serology, reported or confirmed history of prior $\mathrm{CM}$ infection and antifungal therapy information. We also obtained information that included: post-transplantation serology, microbiology and cytology or histopathology reports indicating evidence of CM infection. When present, the site of infection (pulmonary, extra-pulmonary, or both) as well as initiation of antifungal therapy was recorded.

To complement and verify information about CM disease status, we asked our State Health Department office to cross reference our list of cases with CM cases reported to the state since 1997, as some patients could have been diagnosed, tested or treated in other institutions before or after transplant. With all the information required completed, we reviewed our patient list to determine if positive testing results were reported before or after transplantation.

Living status was collected and confirmed though EMR. When the information was not available, we completed the status checking our records and the Social Security office information at the end of our review period (May 31, 2014). When available, cause of death was recorded from EMR.

\section{CM status}

Most liver transplant candidates had CM serology testing performed as part of their pre-transplant evaluation. At the time, there were no set guidelines for post-transplant serology testing and most were performed on a case-by-case basis. Serologic testing, cultures, or histopathology specimens had been obtained when a CM was clinically suspected. Close to half of the patients included in this study had serologic testing performed at least once in the post-transplant period (38 patients out of 78) (Fig. 1).

\section{Definitions}

Positive pre-transplant CM status included reactive or indeterminate serology results by immunoglobulin $\mathrm{G}(\mathrm{IgG})$ or immunoglobulin $\mathrm{M}$ (IgM) antibodies via immunodiffusion, complement fixation, and/or enzyme immunoassay (EIA) methods. A selfreported or physician reported past history of CM were also considered as a positive pre-transplant CM status. Physician documented infections diagnosed by microbiology (culture and DNA probe) or histopathology pre-transplant were also included.

Positive post-transplant CM status included reactive or indeterminate serology by IgG or IgM antibodies via immunodiffusion, complement fixation, and/or or EIA methods, microbiology (culture and DNA probe) and/or histopathology.

\section{CM serology}

The CM serology testing method utilized at our laboratory changed in the middle of 2012. Before 2012, our medical center used the Meridian Bioscience, Inc ${ }^{\circledR}$ test kits, an immunodiffusion method. In 2012, the testing method was changed to EIA by Immy ${ }^{\circledR}$ which was the testing method employed at the time this study was completed. The microbiology and histopathology data that were recorded during data collection were performed at our institution's laboratories.

\section{CM prophylaxis}

Patients were categorized into two groups according to the dose of antifungal agents they received for prophylaxis. The patients were divided into LD prophylaxis group or SD prophylaxis group. The decision to prescribe LD or SD fluconazole was up to prescribing physician. The patients were then further 


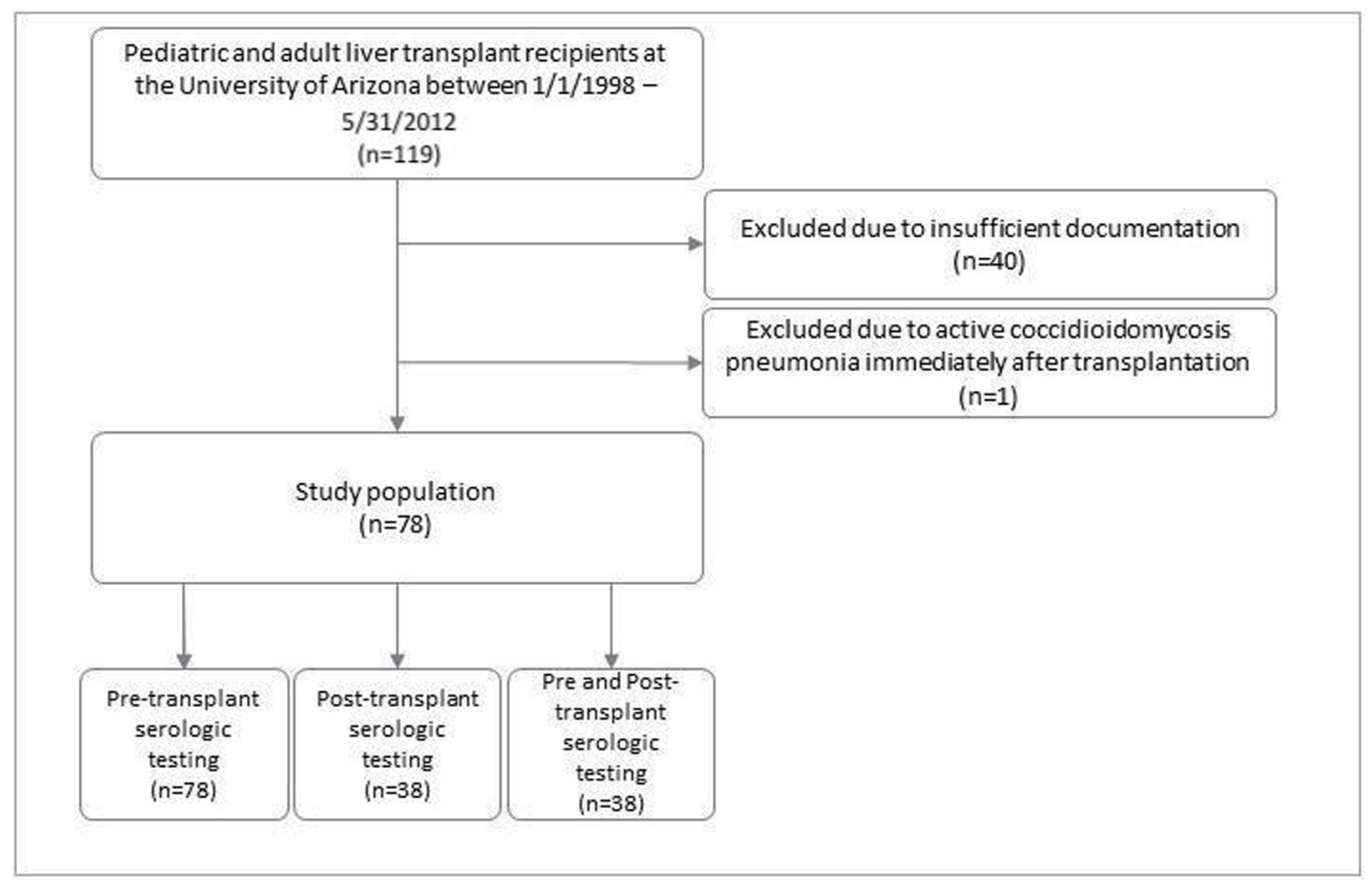

Figure 1. Patient population.

categorized into three groups based on the duration of prophylaxis received post-transplantation (6 months, 12 months, or greater than 12 months).

A positive pre- or post-transplant $\mathrm{CM}$ status incidence rate among liver transplant recipients was calculated from the data collected, and the results were compared between the abovedefined groups.

\section{Immunosuppression}

Use of immunosuppression (including induction therapy) as well as number and choice of maintenance agents was compared among patient groups. Episodes of rejection along with receipt of steroid boluses, thymoglobulin, or monoclonal antibodies were also taken into consideration when analyzing patient immunosuppression status.

\section{Statistical analysis}

Data were analyzed with SPSS version 22 IBM $^{\circledR}$. We used simple descriptive statistics for analysis of the data obtained in the study. Fisher's exact test with two-sided significance was used to assess differences among proportions of various attributes of patients classified according to dosage (LD or $\mathrm{SD})$. Continuous variables were analyzed and compared between the LD and SD groups and between the three duration groups with independent sample $t$-test. Kaplan-Meier survival analysis with log-rank test was used to assess patient survival between LD and SD groups.

\section{Results}

A total of 119 patients underwent liver transplantation surgery between January 1, 1998 and May 31, 2012. All 119 charts were reviewed, 40 were excluded due to insufficient documentation and one was excluded due to having active CM pneumonia diagnosed immediately after transplantation and was placed on antifungal therapy. Out of the 78 patients remaining, 38 were suspected of having CM post-transplant and therefore had post-transplant workup performed. All 78 patients were included in data analysis.

\section{Patient characteristics}

Patient baseline characteristics are summarized in Table 1. There were 11 patients in the SD prophylaxis group, seven of which had renal impairment and achieved SD of fluconazole after adjusting for renal dose, and 67 in the LD prophylaxis group. Patients in the SD prophylaxis group had a mean age of 18.9 years (range: 6 months to 66 years), while patients in the LD prophylaxis group were older with a mean age of 34.3 years (range: 6 months to 68 years), although the difference was not statistically significant. Forty-six percent (46\%) of the patients were male. In about one-third of patients $(28.2 \%)$, infection with hepatitis $\mathrm{C}$ virus was the etiology of end-stage liver disease (ESLD). The mean body mass index (BMI) was 
Table 1. Sample Baseline Demographics

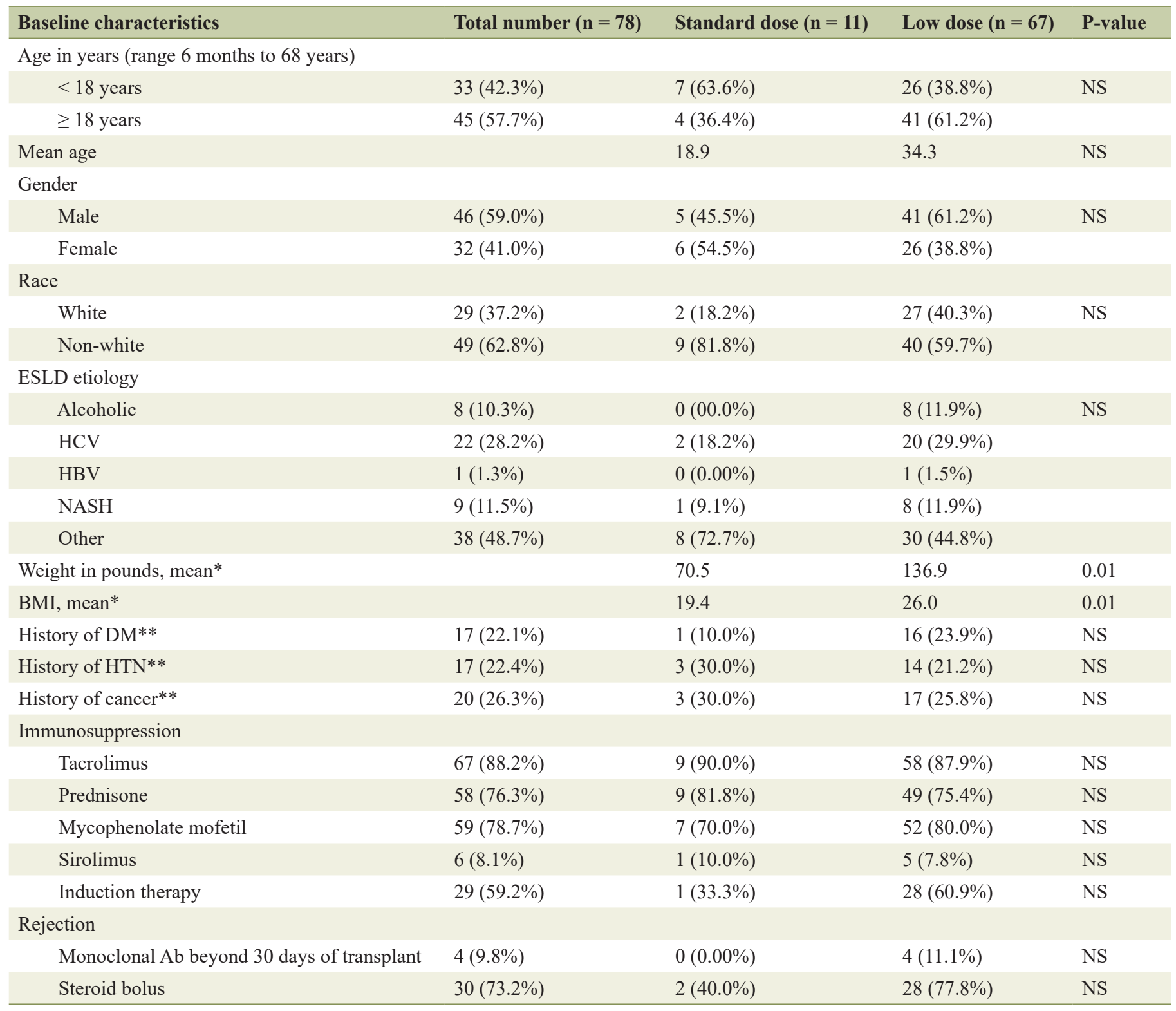

*Weight and BMI calculated before the time of transplant. **Pre-transplant. BMI: body mass index; ESLD: end-stage liver disease; HCV: hepatitis C virus; HBV: hepatitis B virus; NASH: non-alcoholic steatohepatitis; DM: diabetes mellitus; HTN: hypertension; Ab: antibody.

higher in the LD prophylaxis group versus SD group (26.0 vs. $19.4, \mathrm{P}=0.01$ ), which might be explained by the greater patients mean age in the LD prophylaxis group. There were no significant differences between the two groups in terms of other baseline comorbidities. Immunosuppression regimen and dose was similar between the two groups. Rejection was more frequent in the LD prophylaxis group; however, the difference was not statistically significant.

\section{Post-transplant CM status}

Post-transplant CM status analyzed between $\mathrm{SD}$ and LD groups as well as against pre-transplant CM status and duration of prophylaxis are summarized in Figures 2-4. There were more patients with positive post-transplant CM status in the SD group compared to the LD prophylaxis group (Fig. 2 ). When comparing post-transplant status to pre-transplant CM status, we found that more patients with positive pretransplant status had developed reactivation post-transplant (Fig. 3). There was no significant correlation between the three groups classified according to duration of prophylaxis and post-transplant CM status (Fig. 4). However, their primary care physicians or private outpatient specialists may have placed some patients back on prophylaxis. Other variables including demographic information, ESLD etiology, pre-trans- 


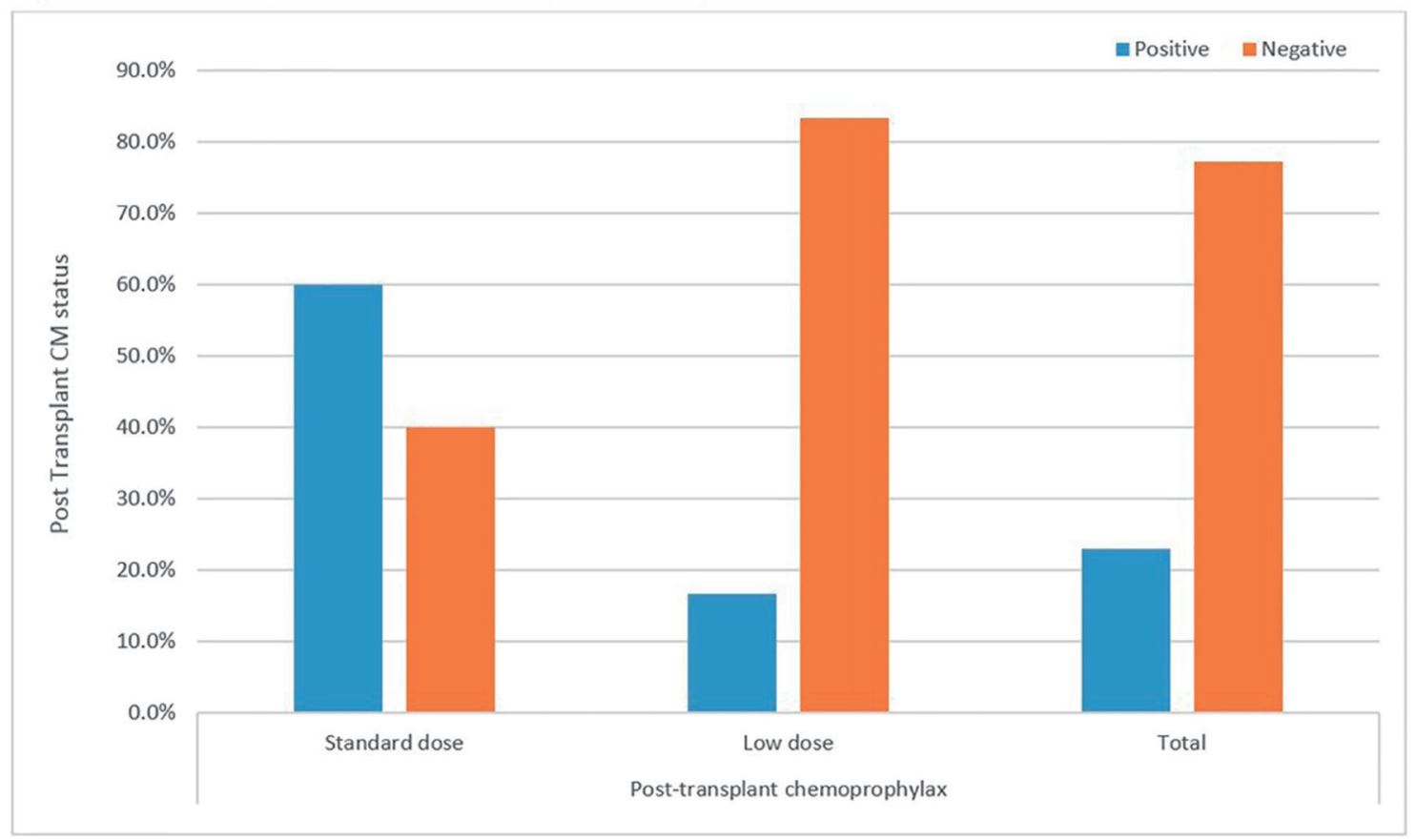

$N=35 P=0.03$ Pearson correlation coefficient 0.33

Figure 2. Post-transplant coccidioidomycosis status compared with prophylaxis dose.

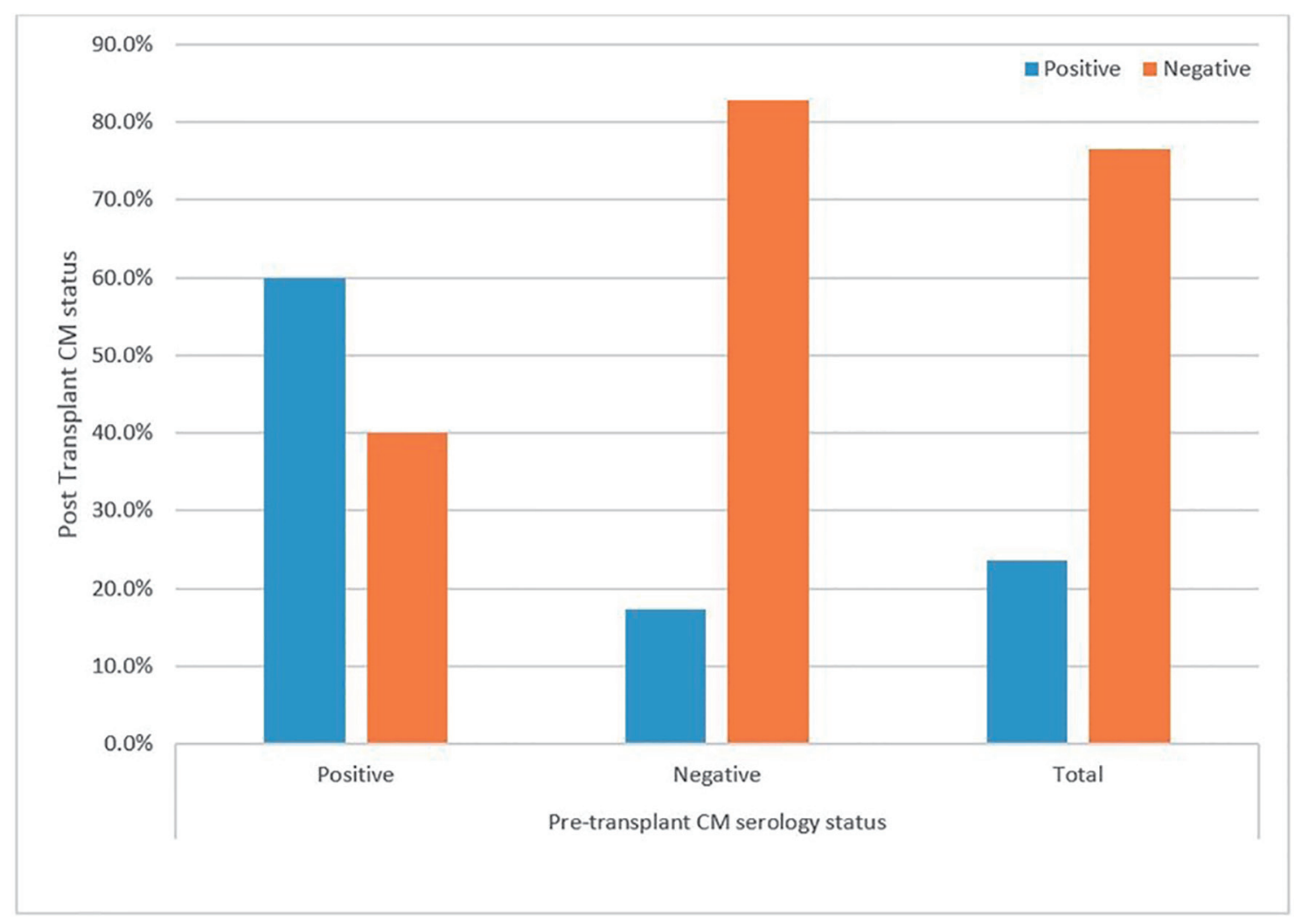

$N=35 P=0.03$ Pearson correlation coefficient 0.35

Figure 3. Post-transplant coccidioidomycosis status compared with pre-transplant status. 


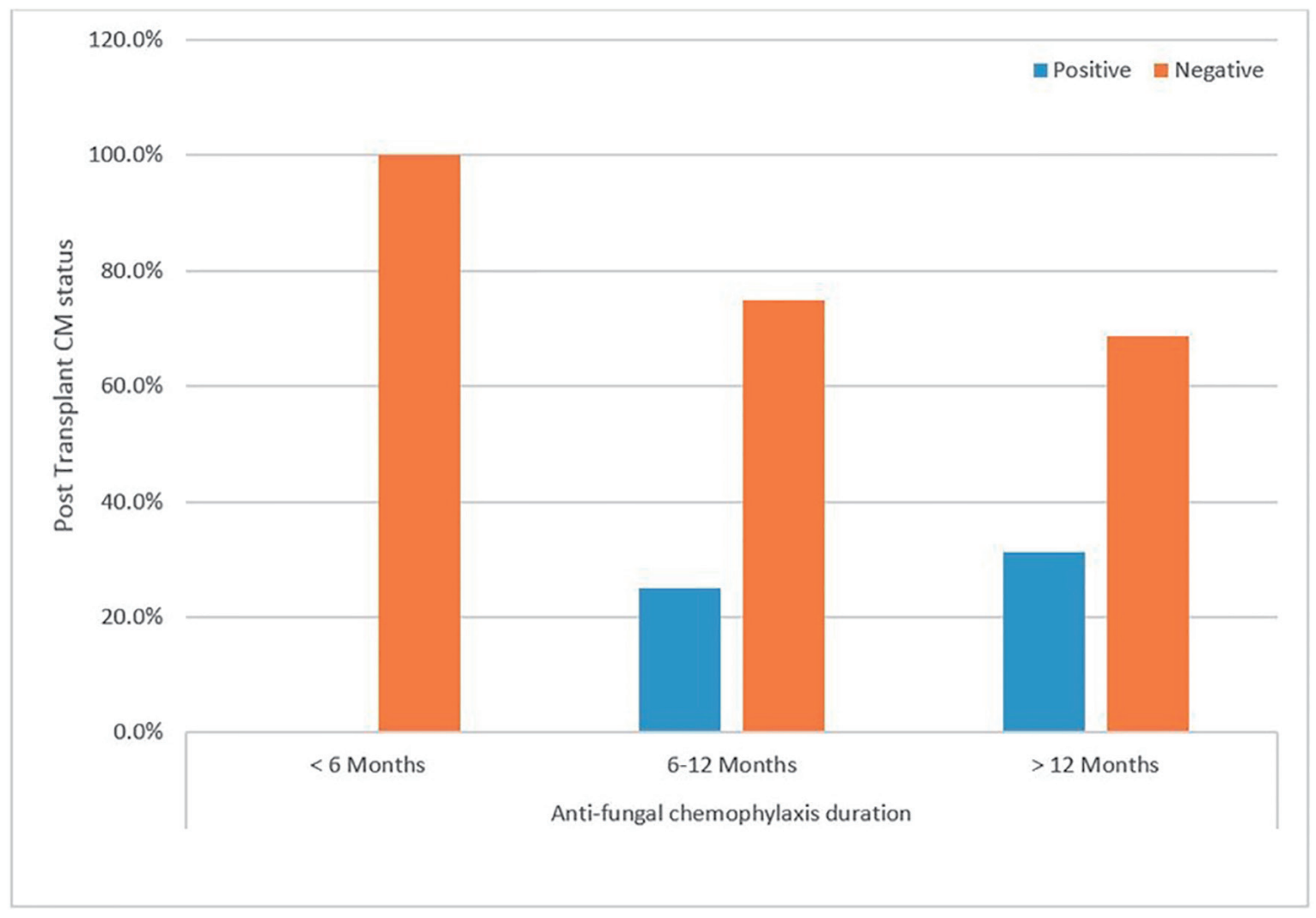

$N=28 P=0.24$ Pearson correlation coefficient 0.22

Figure 4. Post-transplant coccidioidomycosis status compared with prophylaxis duration.

plant comorbid conditions, immunosuppression and rejection were also analyzed in regards to post-transplant CM status, but no significant correlations were found. Using multivariate analysis, a statistically significant correlation was found between positive pre-transplant status and positive post-transplant status with a hazards ratio of 8.25 (95\% confidence interval (CI): 1.028 - 66.192).

\section{Survival analysis}

There was a trend towards improved survival in patients who had a positive post-transplant CM status in the SD group versus the LD group (90.9\% versus $81.3 \%$ ) (Fig. 5). However, this was not statistically significant. Survival was lower in patients who were unchecked for post-transplant CM infection, suggesting underestimation of detection (Fig. 6). The difference was not statistically significant.

\section{Individual patient analysis}

Given that our study had a small number of patients $(\mathrm{N}=78)$, we were able to perform more in-depth analysis of individual charts of patients with either positive pre-transplant (eight patients) and/or positive post-transplant (nine patients) CM status.
Out of the 38 patients who were checked for post-transplant CM status, nine $(23.7 \%)$ were positive. One patient developed disseminated infection while on LD prophylaxis 2 years posttransplant. Two patients developed pulmonary CM: one 4 months while on LD prophylaxis and the other 1 year after completion of LD prophylaxis. Two patients had asymptomatic serologic conversion after completion of LD prophylaxis. The first conversion was detected 6 months after completion and the second a year after finishing treatment. There were three patients who were positive pre-transplant and remained seropositive post-transplant but remained asymptomatic (one was in SD group and two were in LD group). One patient seroconverted to asymptomatic positive post-transplant CM status after completion of 6 months of SD prophylaxis. Out of the total 78 included patients, seven patients $(8.9 \%)$ were positive pre-transplant, of which six were placed on LD and one was on SD prophylaxis. For those six placed on LD prophylaxis, four converted to negative serology post-transplant and remained asymptomatic until the end of chart review period. The other two patients on LD prophylaxis remained seropositive and asymptomatic. The one patient placed on SD prophylaxis remained seropositive and asymptomatic.

There was also one patient (excluded from the analysis) who underwent transplantation with $\mathrm{CM}$ pneumonia. He had been placed on SD but died 2 months after transplant from complications of anoxic brain injury caused by hypoxia. Current practice protocol based on expert recommendation would make 


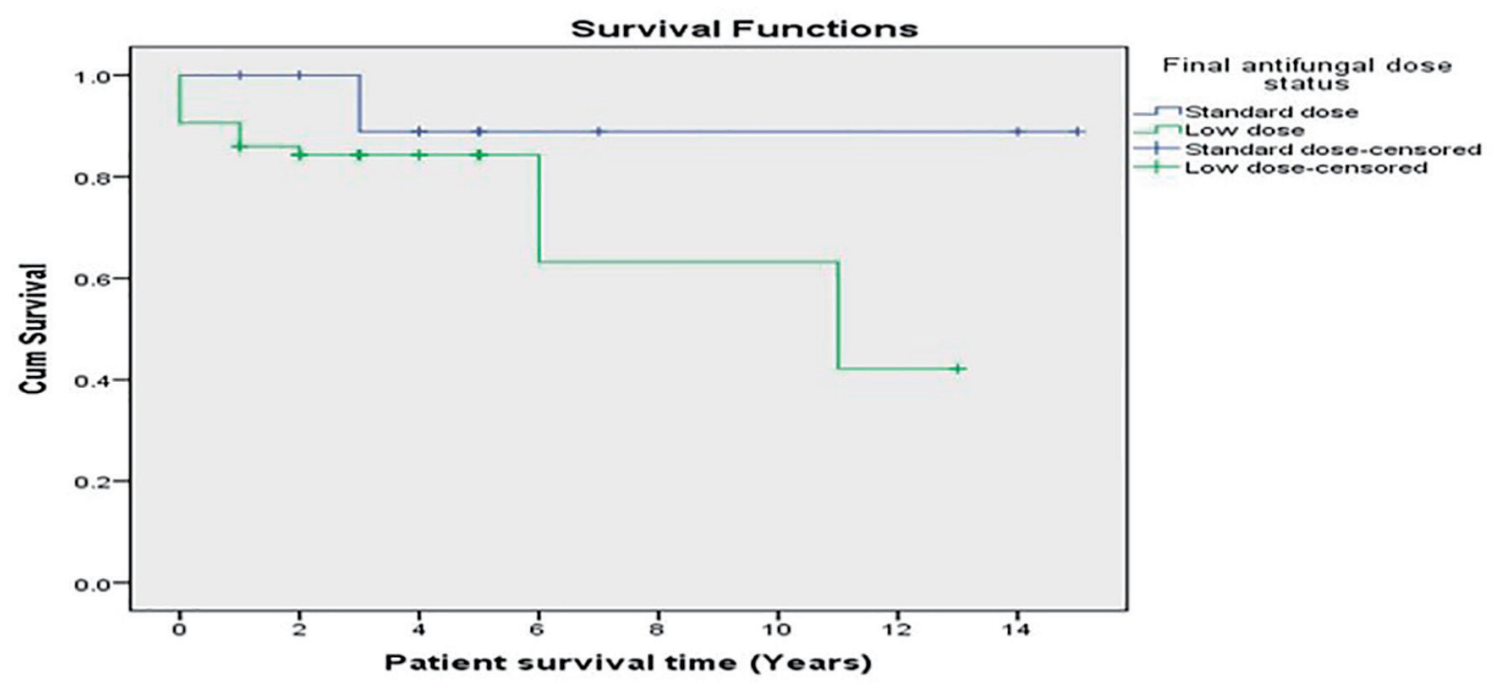

\begin{tabular}{|c|c|c|c|c|}
\hline $\begin{array}{c}\text { Final antifungal } \\
\text { dose status }\end{array}$ & Total N & N of Events & N & Percent \\
\hline Standard dose & 11 & 1 & 10 & $90.9 \%$ \\
\hline Low dose & 64 & 12 & 52 & $81.3 \%$ \\
\hline Overall & 75 & 13 & 62 & $82.7 \%$ \\
\hline
\end{tabular}

Figure 5. Survival based on antifungal prophylaxis dose.

any active $\mathrm{CM}$ a contraindication to organ transplantation.

\section{Discussion}

Coccidioides immitis/posadasii is associated with life-threatening infections among transplant recipients. The risk of infection among SOT recipients in endemic regions in the absence of prophylaxis may reach $9 \%$ in the first year after transplantation [2]. SOT recipients experience a high rate of disseminated infection. Therefore, most transplant centers in the endemic regions have developed their own prophylaxis protocols. Previously, all transplant recipients at the University of Arizona Medical Center, now Banner University Medical Center, received fluconazole at a dose of $50 \mathrm{mg}$ daily for an indefinite period of time for prophylaxis. It is unclear as to when or why this particularly low dose was chosen at our institution, but the practice was called into question when several kidney and heart transplant recipients developed breakthrough infections while on $50 \mathrm{mg}$ daily dose.

In our study, there were more patients with a positive post-transplant CM status in the SD group compared to the LD prophylaxis group. This may point to a possible hidden bias where the clinicians at the time may have purposely increased or prescribed the standard dose for these patients. Unfortunately, we could not find this information in the charts. Additionally, one could speculate that the standard dose had been protective in this group. We also found more patients with a positive pre-transplant CM status that developed infection post-transplant. We found a statistically significant correlation between positive pre-transplant $\mathrm{CM}$ status and positive post-transplant CM status, which highlights the importance of pre-transplant status as a predictor of future post-transplant infection.

We detected a trend towards improved survival (SD group $90.9 \%$ versus LD $81.3 \%$ ) among those with a positive post-transplant CM status. However, this survival difference was not statistically significant. The lack of significance may have been due to low power impact and data that could not be located. In addition, the difference found might have been due to other infections such as those caused by candida species, which respond to fluconazole. Despite the information written in the charts we also became aware that many of the patients continued higher dose of prophylaxis prescribed by community physicians. We found no significant correlation between duration of prophylaxis and post-transplant CM status; however, it is likely that their primary care physicians or private outpatient specialists may have placed some patients back on prophylaxis, as this is a common practice in Arizona after patients return to the community. Another important factor to highlight is the decreased sensitivity of serologic testing in SOT. Serological antibody testing for CM is useful but may provide false negative results in immunosuppressed patients. 


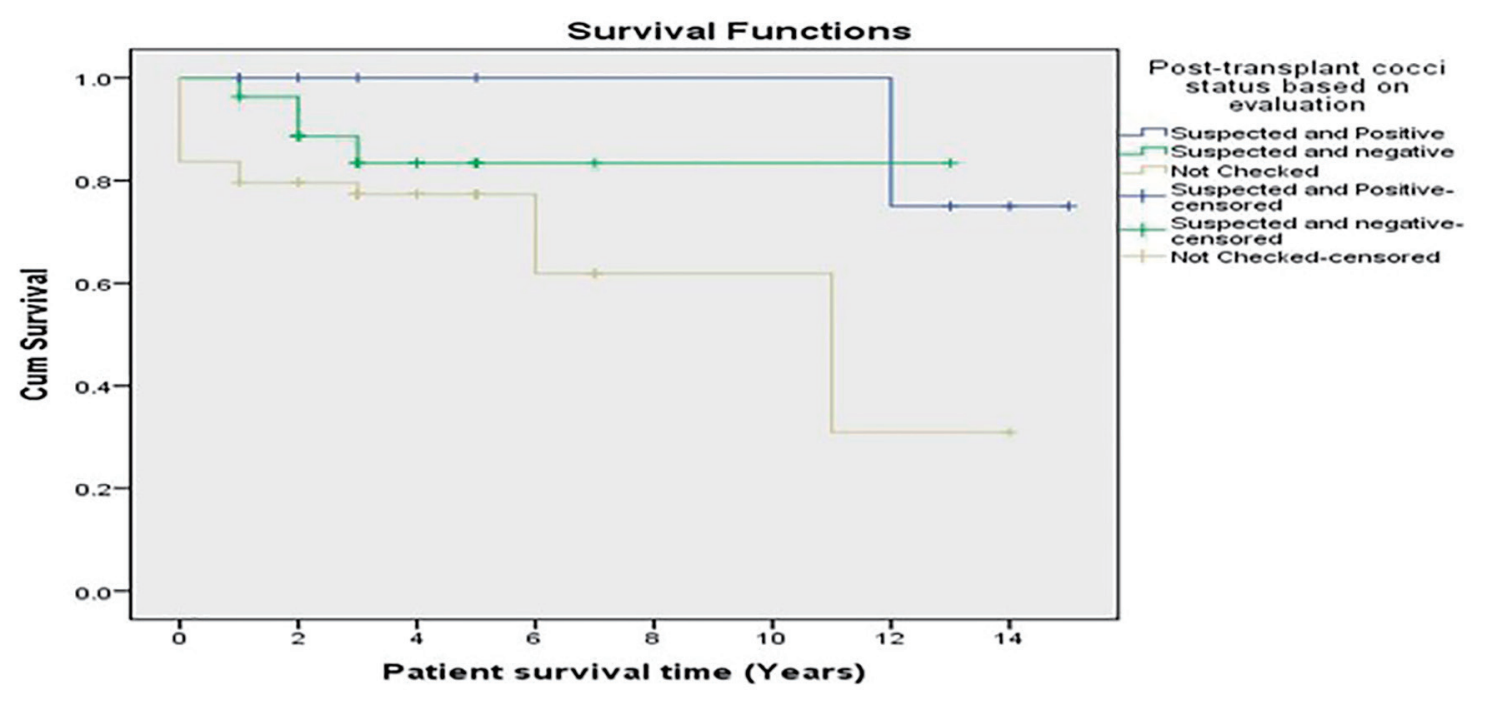

\begin{tabular}{|c|c|c|c|c|}
\hline $\begin{array}{c}\text { Post-transplant cocci } \\
\text { status based on } \\
\text { evaluation }\end{array}$ & Total N & N of Events & N & Percent \\
\hline Suspected and Positive & 9 & 1 & 8 & $88.9 \%$ \\
\hline $\begin{array}{c}\text { Suspected and } \\
\text { negative }\end{array}$ & 27 & 4 & 23 & $85.2 \%$ \\
\hline Not Checked & 49 & 13 & 36 & $73.5 \%$ \\
\hline Overall & 85 & 18 & 67 & $78.8 \%$ \\
\hline
\end{tabular}

Figure 6. Survival based on coccidioidomycosis evaluation post-transplant.

Blair and colleagues reported the positivity for any single serologic test ranging from $21 \%$ to $56 \%$ in these populations, which may have impacted the results of our study [5].

Distinction between asymptomatic and active infection was challenging based on chart reviews and certain patients had received their care at other institutions, including the group that had their testing results reported to the health department. Survival was lower in patients who were unchecked for posttransplant CM suggesting underestimation of detection but the difference was not statistically significant. Despite the lack of statistical significance in the current study, we have changed our protocol and currently use universal prophylaxis for 12 months with SD of fluconazole (200 mg daily with renal dose adjustment) post-transplant with the exception of liver transplant recipients who receive universal prophylaxis for only 6 months to avoid risk of associated hepatotoxicity. Since the institution of SD prophylaxis at our institution, we have not diagnosed further new post-transplant infections. We also utilize the proposed prophylaxis by Blair et al where patients with a history of recent CM or positive serologic results during the period of transplantation receive fluconazole at $200 \mathrm{mg}$ /day for life [6].

We understand that our study may not have had the number of subjects needed to show significance between SD and LD prophylaxis. A large prospective randomized clinical trial would best answer the questions raised in this study. Finally, patients undergoing liver transplantation tend to receive lower doses of immunosuppression; therefore, a larger study that includes renal, lung and heart transplant recipients may provide even better results.

\section{Acknowledgments}

Thanks are given to Orion McCotter, MPH and Farah Alsafar, BS.

\section{Financial Disclosure}

No relevant financial disclosures.

\section{Conflict of Interest}

The only conflict of interest to disclose is Dr. Tirdad Zangeneh has a small grant from Astellas and a grant from Shire Pharmaceuticals. None of the other authors have a conflict of interest 
to disclose.

\section{Informed Consent}

The University of Arizona Institutional Review Board (IRB) granted a waiver of informed consent requirement.

\section{Author Contributions}

Dr. Habib is the first author. Dr. Zangeneh is the senior author and co-authored the manuscript. El Ramahi co-authored the manuscript. Dr. Casal co-authored the manuscript and along with Mr. McCotter reviewed data from the Arizona Department of Health Services data base. Dr. Rosen, Dr. Farran, Dr. Shubeilat and Dr. Walker assisted with chart review and data collection. Ms. Alsafar assisted with manuscript review and submission.

\section{Abbreviations}

EMR: electronic medical records; SD: standard dose; LD: low dose; SOT: solid-organ transplant; IDSA: Infectious Diseases Society of America; CM: coccidioidomycosis; IRB: Institutional Review Board; IgG: immunoglobulin G; IgM: immunoglobulin M; EIA: enzyme immunoassay

\section{References}

1. Arizona Department of Health Services: valley feverreports \& publications [internet]. Cited 2016. Nov 27. Available from: http://www.azdhs.gov/documents/preparedness/epidemiology-disease-control/valley-fever/reports/valley-fever-2015.pdf.

2. Blair JE. Coccidioidomycosis in patients who have undergone transplantation. Ann N Y Acad Sci. 2007;1111:365376.

3. Galgiani JN, Ampel NM, Blair JE, Catanzaro A, Geertsma F, Hoover SE, Johnson RH, et al. Executive summary: 2016 Infectious Diseases Society of America (IDSA) clinical practice guideline for the treatment of coccidioidomycosis. Clin Infect Dis. 2016;63(6):717722.

4. Kahn A, Carey EJ, Blair JE. Universal fungal prophylaxis and risk of coccidioidomycosis in liver transplant recipients living in an endemic area. Liver Transpl. 2015;21(3):353-361.

5. Blair JE, Coakley B, Santelli AC, Hentz JG, Wengenack NL. Serologic testing for symptomatic coccidioidomycosis in immunocompetent and immunosuppressed hosts. Mycopathologia. 2006;162(5):317-324.

6. Vucicevic D, Carey EJ, Blair JE. Coccidioidomycosis in liver transplant recipients in an endemic area. Am J Transplant. 2011;11(1):111-119. 